\title{
Temperature Variation of Mass Spectra of Hydrocarbons ${ }^{1}$
}

\author{
Robert M. Reese, Vernon H. Dibeler, and Fred L. Mohler
}

\begin{abstract}
The temperature variations of the mass spectra of a variety of hydrocarbons have been measured by using a Consolidated mass spectrometer. Measurements have been made in the temperature range of $100^{\circ}$ to $300^{\circ} \mathrm{C}$ on a number of selected mass peaks in the spectra of 21 compounds. The sensitivity (current per unit pressure) of the maximum peak and the ratio of other peaks to the maximum peak have been measured as a function of temperature of the ion source. The percentage change of sensitivity or of pattern per degree $\mathrm{C}$ at $250^{\circ} \mathrm{C}$ has been defined as the temperature coefficient. This changes very little with temperature. The temperature coefficients of sensitivity for $\mathrm{A}$ and $\mathrm{N}_{2}$ are -0.23 and -0.18 . Sensitivity coefficients of the maximum peaks of the hydrocarbons range from -0.2 to 0 ; and pattern coefficients of most of the peaks have small negative or positive values, but the molecule ions have larger negative coefficients up to -1.5 percent per degree. There is an inverse correlation between the size of the molecule ion peak relative to the sum of all the peaks and the temperature coefficients. Spontaneous dissociation of molecule ions because of thermal energy can account for this correlation between peak height and temperature coefficient. Published appearance potential data for a few of these molecules give a basis for computing the stability of the ions and show that thermal dissociation is to be expected at $200^{\circ}$ to $300^{\circ} \mathrm{C}$.
\end{abstract}

\section{Introduction}

In 1944, Hipple [1] ${ }^{2}$ reported a marked temperature effect on the relative abundance of various ions in mass spectra of hydrocarbons. In a subsequent note, Fox and Hipple [2] briefly described the effects of temperature on normal and isobutane and 2,2,3trimethylpentane and pointed out that such effects were useful as a means of measuring the temperature of the ion source. More recently, Stevenson 3] has reported the temperature effects on a number of simple molecules and has discussed the theoretical reasons for the existence of such effects. Berry [4] has examined the effect of temperature on the mass spectra of nine saturated and seven unsaturated hydrocarbons. These experiments show that for monatomic gases and simple, stable molecules there is a relatively small decrease in ion current per unit of pressure with increasing temperature, arising from the decrease in gas density and from a decrease in the efficiency of collecting ions of higher initial kinetic energies. In the hydrocarbons, the molecule ions and some fragment ions show a temperature effect of a much larger magnitude. The decrease in the molecule ion is greater than for any of the fragment ions, and Stevenson [3] finds that an inverse correlation exists between the temperature effect and the fraction of total ionization contributed by the molecule ion.

This temperature effect is a phenomenon of importance in understanding the mechanism of ionization of polyatomic molecules, and it is of a magnitude sufficient to cause large errors in mass spectrometric analysis if the ionization chamber is not held at a constant temperature. For these reasons, it has seemed worth while to extend the research on temperature effects to a wide variety of hydrocarbons.

\footnotetext{
${ }^{1}$ A preliminary report of this work was made at the New York meeting of the A merican Physical Society, February 1950.

${ }^{2}$ Figures in brackets indicate the literature references at the end of this paper.
}

\section{Experimental Details}

A Consolidated model 21-102 mass spectrometer, equipped with a thermostatted ionization chamber and automatic control of the electron emission was used to measure the effect of temperature on mass spectra. The leads of the thermocouple that normally operates the thermostatic control were connected to a potentiometer in order to measure the temperature of the ion source. With the filament off and the source at room temperature, a sample was introduced and the filament was raised to full emission as rapidly as possible. The relative abundance of a selected group of ions was repeatedly recorded at constant magnetic field as the temperature increased to about $200^{\circ} \mathrm{C}$ due to the heat of the filament. At this point the external heat lamp was turned on, and data were recorded to over $300^{\circ} \mathrm{C}$. As each mass peak was recorded, the temperature was read. Berry [4] has stated that the gas is in thermal equilibrium with its surroundings before ionization takes place. The gas temperature is assumed to be some function of the temperature measured by the thermocouple. Total elapsed time was also recorded, and periodic readings of the sample pressure in the gas reservoir were made with an indicating micromanometer [5].

A typical temperature-time curve for the ion source is shown in figure 1. For this ion source a maximum temperature of $250^{\circ} \mathrm{C}$ was reached in about 1 hour. Radiation from the external heat lamp raised the temperature an additional $75^{\circ}$. If the heat lamp was turned on at $200^{\circ} \mathrm{C}$, a temperature of $300^{\circ} \mathrm{C}$ was reached in about $40 \mathrm{~min}$, and during this time the selected ion peaks were successively recorded as rapidly as possible. One or more mass peaks in each of two to six carbon groups were recorded. In general, only the principal mass peaks in addition to the molecule ion were recorded. 
The hydrocarbons used were NBS standard samples with a few exceptions. Complete mass spectra of all of the compounds have been published [6] along with information on the source and purity of the compounds. Nitrogen containing approximately 65 atom percent of $\mathrm{N}^{15}$ was obtained from Eastman Kodak Co., and argon was obtained from Air Reduction Corp.

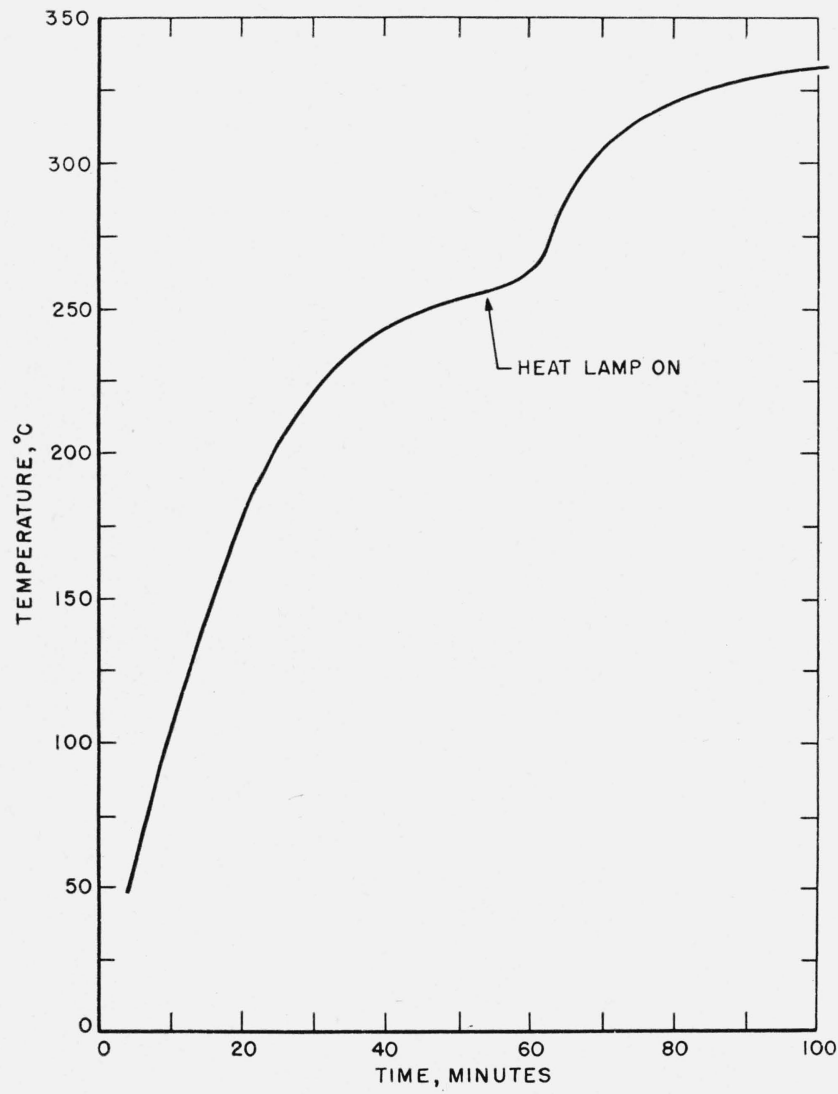

Figure 1. Temperature rise of the ionization chamber as a function of time.

As a test of the method of measurement, we have repeated the measurements of Stevenson [3] on argon and nitrogen and the measurements of Berry [4] on $n$-butane and two of the octanes. There is satisfactory agreement, and our method of measuring the temperature effect on spectra without waiting for temperature equilibrium seems to be entirely valid. This is important as it permits studying spectra of many compounds without prohibitive interruption of other research and testing programs.

\section{Experimental Results}

Figure 2 is a plot of the data obtained for 12 peaks of $n$-octane at different temperatures. Ordinates give the sensitivity of the base peak of mass 43 and the pattern ratio for all other ions. ${ }^{3}$ When the

3 The following definitions are used: The sensitivity is the ion current per unit pressure in the gas reservoir, the base peak is the maximum peak in the spectrum, and the pattern ratio is the intensity of any other peak relative to the base peak. pattern ratios and sensitivities are plotted on a logarithmic scale against the measured temperature, the resulting semilogarithmic plots are nearly

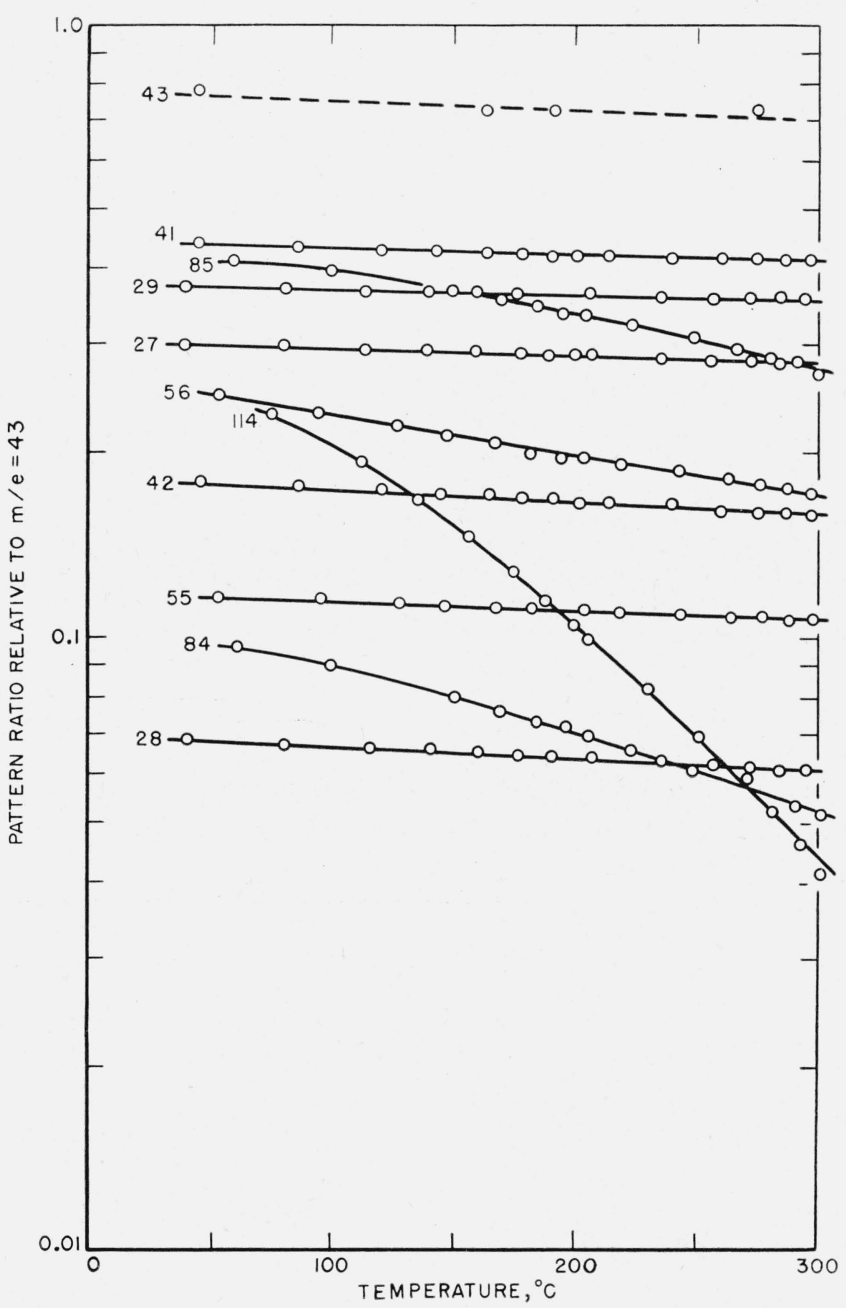

Figure 2. Plot of the effect of temperature on the pattern ratios of selected peaks of $n$-octane.

The ordinate for the 43 pcak is in terms of sensitivity (divisions per micron) with a scale 100 times that indicated.

straight lines [4] and the slope of these curves at $250^{\circ} \mathrm{C}$ in units of percentage change per degree centigrade has been taken as the temperature coefficient for the sensitivity and for the pattern ratio. The temperature coefficient for the sensitivity of any ion other than the base peak is equal to the temperature coefficient of the base peak plus the temperature coefficient of the pattern ratio. A temperature of $250^{\circ} \mathrm{C}$ has been selected as appropriate to the range of normal operating temperatures for the Consolidated mass spectrometer. However, most of the curves are nearly linear, and, in general, the pattern ratios and sensitivities change very slowly with temperature. Berry has used a similar method of evaluating temperature coefficients but has computed values at $160^{\circ} \mathrm{C}$. 
TABLE 1. Temperature coefficients of pattern coefficients and of sensitivity for paraffin hydrocarbons ${ }^{\mathrm{a}}$

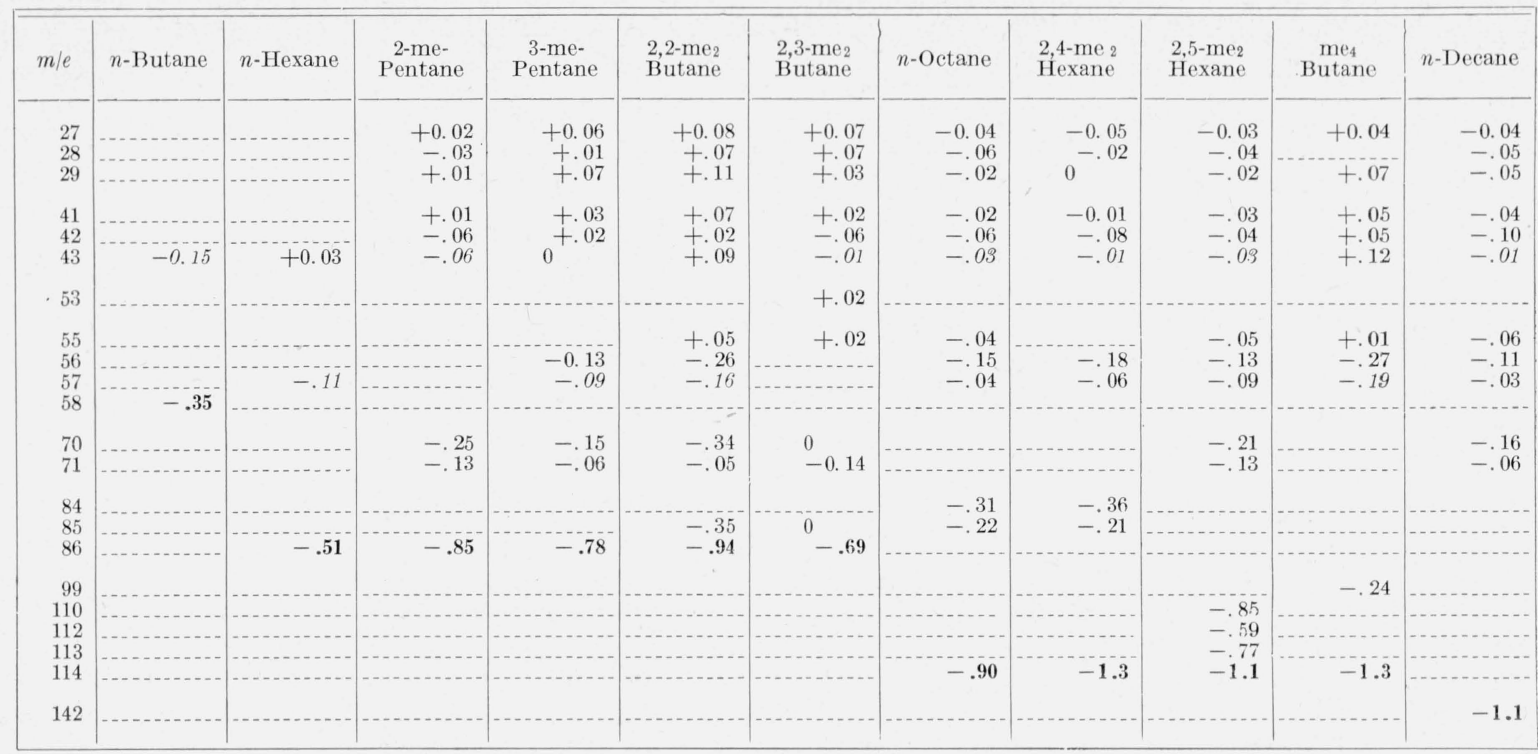

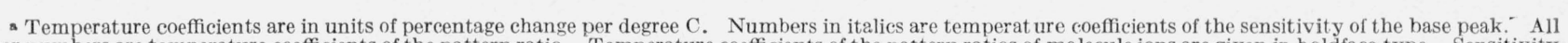

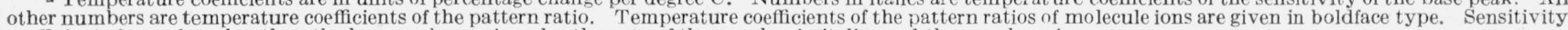
coefficients for peaks other than the base peak are given by the sum of the number in italics and the number given.

TABLE 2. Temperature coefficients for mass spectra of miscellaneous hydrocarbons and other gases ${ }^{\mathrm{a}}$

\begin{tabular}{|c|c|c|c|c|c|c|c|c|c|c|}
\hline$m / e$ & 1-Hexene & 1-Octene & 1-Decene & Propyne & $\begin{array}{l}\text { 4-me-1- } \\
\text { Pentyne }\end{array}$ & 1-Octyne & $\begin{array}{l}\text { Cyclo- } \\
\text { Hexane }\end{array}$ & Benzene & $\mathrm{N}^{14} \mathrm{~N}^{15}$ & $\mathrm{~A}$ b \\
\hline $\begin{array}{l}26 \\
27\end{array}$ & +0.01 & -0.04 & 0 & & +0.09 & () & 0 & $\begin{array}{r}+0.09 \\
+.07\end{array}$ & & \\
\hline $\begin{array}{l}28 \\
29\end{array}$ & $\begin{array}{c}0 \\
-0.01\end{array}$ & $\begin{array}{l}-.04 \\
-.03\end{array}$ & $\begin{array}{l}-0.02 \\
0\end{array}$ & & $\begin{array}{r}+.08 \\
+.08 \\
+.02\end{array}$ & $\begin{array}{c}0 \\
+0.02\end{array}$ & $\begin{array}{r}+0.04 \\
+\quad .01\end{array}$ & & -0.18 & \\
\hline 39 & & & & 0 & +.04 & & & +.07 & & \\
\hline 40 & & & & -0.14 & +.03 & & & & & -0.28 \\
\hline 41 & -.05 & $\begin{array}{l}-.03 \\
-.02\end{array}$ & $\begin{array}{r}-0.01 \\
+01\end{array}$ & & +.06 & +.01 & +.01 & & & \\
\hline $\begin{array}{l}42 \\
43\end{array}$ & $\begin{array}{l}-.07 \\
-.03\end{array}$ & $\bar{T}^{-.02}$ & $\begin{array}{l}+.01 \\
+.04\end{array}$ & & $\begin{array}{r}-.01 \\
+.07\end{array}$ & $\begin{array}{l}-.02 \\
-.10\end{array}$ & $\begin{array}{l}+.01 \\
+.01\end{array}$ & & & \\
\hline 50 & & & & & & & & +.07 & & \\
\hline 51 & & & & & & $\cdots$ & $\cdots$ & $\begin{array}{r}+.03 \\
\end{array}$ & & \\
\hline $\begin{array}{l}52 \\
53\end{array}$ & & & & & & & 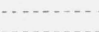 & & & \\
\hline 54 & & & & & +.02 & & & & & \\
\hline $\begin{array}{l}55 \\
56\end{array}$ & $\begin{array}{l}-.06 \\
-.09\end{array}$ & $\begin{array}{r}-0.03 \\
-.10\end{array}$ & & & & & $\begin{array}{l}-.02 \\
-.065\end{array}$ & & & \\
\hline 57 & & -.12 & & & & & & & & \\
\hline 67 & & & & & -.11 & & -.03 & & & \\
\hline $\begin{array}{l}69 \\
69\end{array}$ & -.21 & -.10 & +.05 & & & & $\begin{array}{l}-.10 \\
-.06\end{array}$ & & & \\
\hline 70 & -.20 & -.21 & -.09 & & & & & & & \\
\hline 76 & & & & & & & & & & \\
\hline $\begin{array}{l}77 \\
78\end{array}$ & & & & & & & & +.04 & & \\
\hline 79 & & & & & & -.05 & & & & \\
\hline 80 & & & & & 05 & $\begin{array}{l}-.23 \\
-19\end{array}$ & & & & \\
\hline $\begin{array}{l}11 \\
82\end{array}$ & & & & & $\begin{array}{l}-.97 \\
-.97\end{array}$ & $\begin{array}{l}-.19 \\
-.25\end{array}$ & & & & \\
\hline $\begin{array}{l}83 \\
84\end{array}$ & -41 & $\begin{array}{l}-.21 \\
-.23\end{array}$ & $\begin{array}{l}-.18 \\
-.26\end{array}$ & & & & $\begin{array}{l}-.09 \\
-.92\end{array}$ & & & \\
\hline 95 & & & & & & -.2 & & & & \\
\hline & & & & & & & & & & \\
\hline 112 & & -.83 & & & & & & & & \\
\hline 140 & & & -1.2 & & & & & & & \\
\hline & & & & & & & & & & \\
\hline
\end{tabular}

a See footnote to table 1 .

b $\mathrm{A}^{++}, m / e=20$, has a temperature coefficient of +0.04 for the pattern ratio and of -0.19 for the sensitivity

As shown in figure 2, most of the ions give a small negative slope, whereas the molecule ion $(m / e=114)$ has a large slope of -0.9 percent per degree. There is a fivefold change in the relative intensity of this peak in the temperature range of $100^{\circ}$ to $300^{\circ} \mathrm{C}$.
Tables 1 and 2 give temperature coefficients for all of the mass peaks measured in the hydrocarbons, nitrogen and argon. These values have been read from plots like that shown in figure 2 for $n$-octane. Values of the temperature coefticient of the sensi- 
tivity are given for the base peak and are printed in italics. Other values pertain to the temperature coefficient of the pattern ratio. Coefficients of the pattern ratio for molecule ions are printed in boldfaced type, excepting four compounds the molecule ions of which are also the most abundant ions. For these compounds, values of the sensitivity coefficients are given and are printed in italics.

A change in the pattern corresponding to a temperature coefficient of 0.01 percent per degree is measureable and probably significant for the larger peaks. The uncertainty in the measurement of the temperature coefficient of sensitivity is several times as large as this. Some of the molecule ion peaks are very small, and their temperature coefficients are subject to an uncertainty of at least 10 percent of the value given. In 1-octyne the molecule ion peak at $m / e=110$ is almost entirely accounted for by the $\mathrm{C}^{13}$ isotope of the 109 peak, $\mathrm{C}_{8} \mathrm{H}_{13}{ }^{+}$. Consequently, the molecule ion is immeasurably small.

The nitrogen measurements were made on a mixture of $\mathrm{N}^{14}$ and $\mathrm{N}^{15}$ in which the $\mathrm{N}^{14} \mathrm{~N}^{15}$ molecule was the most abundant. The temperature coefficient is probably not significantly different from that of ordinary nitrogen. The experiment was made to investigate a possible temperature effect on the patterns of isotopic nitrogen molecules, but the results were negative.

In tables 1 and 2 , compounds have been divided into paraffins and other hydrocarbons for convenience in tabulation only. We see no significant difference in temperature coefficients between saturated and unsaturated compounds. In all hydrocarbons, as in $n$-octane, the molecule ion has the largest temperature coefficient, and in most cases it has by far the largest value with a range of values from -1.3 to -0.14 . Temperature coefficients for the sensitivity of the base peak have small values ranging from 0 to -0.2 . Pattern ratios sometimes have small positive temperature coefficients of +0.1 or less.

The peak at $m / e=110$ in 2,5-dimethylhexane is the result of a metastable transition arising from a molecule ion of mass 114 , which loses two hydrogen atoms after traversing the ion accelerating field to give an ion with an apparent mass of approximately 110 rather than 112 . One would expect a higher energy level for the excited state corresponding to the metastable dissociation. As the temperature coefficient of the 110 peak is definitely less than that of the 114 peak, it would appear reasonable that increased temperature results in increased probability of excitation to the metastable state.

Table 3 summarizes the temperature coefficients of pattern ratio and of sensitivity for all of the molecule ions in tables 1 and 2. For most of the molecules the two coefficients are rearly equal, as the temperature coefficient of sensitivity of the base peak is relatively small. Stevenson [3] has pointed out that there is an inverse correlation between the temperature effect and the fraction of total ionization contributed by the molecule ion. The last column in table 3 gives this fraction for the various mole- cules. The fraction is computed from published spectra [6].

TABLE 3. Temperature coefficients for pattern and sensitivity of molecule ions in Percentage per degree at $250^{\circ} \mathrm{C}$

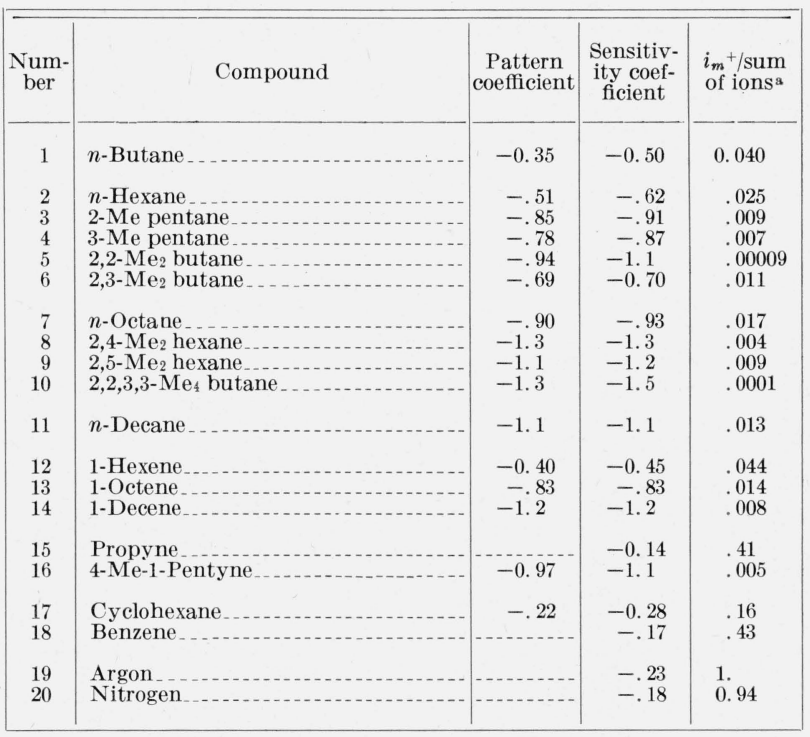

a Ratio of molecule ion peak to sum of mass peaks at $250^{\circ} \mathrm{C}$.

Figure 3 is a plot of the data in the last two columns of table 3 showing the correlation between the temperature coefficient of sensitivity and the reciprocal of the ratio in the last column of table 3 . Ordinates give the logarithm of the ratio, and abscissae give the temperature coefficient. The compounds are identified by referring to the index numbers in table 3 . Except for two of the points there is a good correlation between the two quantities plotted. The two compounds that give points far above the curve, 2,2-dimethylbutane and 2,2,3,3tetramethylbutane, have very small molecule ion peaks; and although the measurements are quite inaccurate, the evidence is fairly convincing that the curve cannot be extrapolated to very small values of the ratio of molecule ion to total ionization.

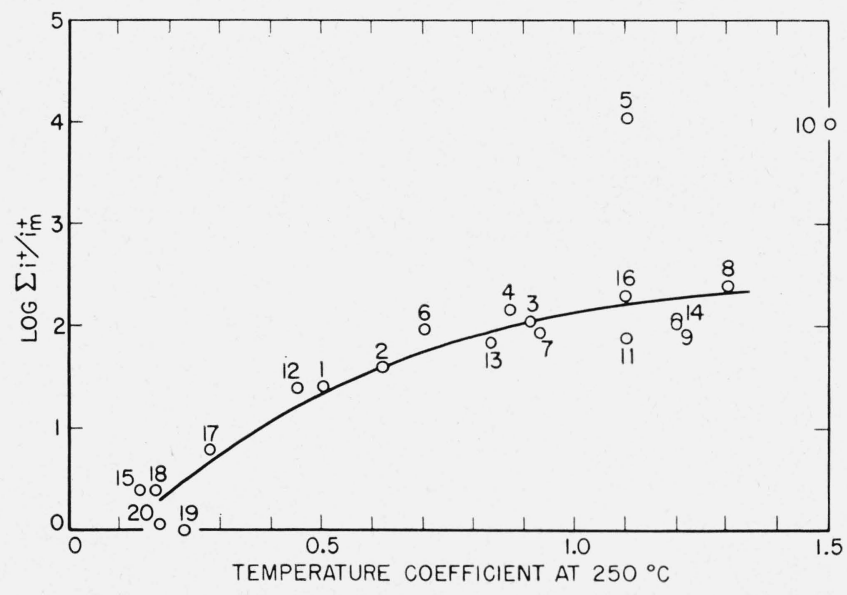

Figure 3. Correlation of the temperature coefficient of the molecule ion with the fraction of total ionization contributed by the molecule ion. 


\section{Discussion of Results}

These measurements are in satisfactory agreement with published results on temperature effects. Stevenson's measurements [3] indicate values of -0.21 for the temperature coefficient of sensitivity of argon and -0.18 for nitrogen. (These values are based on data read from published curves). Our values were -0.23 and -0.18 , respectively. Berry's [4] values for the molecule ion of $n$-butane (at $160^{\circ} \mathrm{C}$ ) were -0.3 for the coefficient of the pattern ratio and -0.6 for the coefficient of the sensitivity. Our values (table 3 ) were -0.35 and -0.50 , respectively. His values for 2,4-dimethylhexane are -1.0 and -1.4 , as compared with values of -1.3 for both coefficients in table 3 . Berry's values for temperature coefficients of other mass peaks in this spectrum and in 2,2,3,3-tetramethylbutane differ in detail however from data in table 1 .

The sensitivity for monatomic gases and stable molecules will decrease with increasing temperature because of the decrease in gas density, and because the collecting efficiency of the mass spectrometer decreases with increasing kinetic energy of the ions. Stevenson [3] has shown that the temperature effects for the rare gases, $\mathrm{N}_{2}$ and $\mathrm{CO}_{2}$ conform to theory. The gas density effect will give a temperature coefficient of -0.10 percent per degree, and the collecting efficiency will decrease by an amount that depends on the instrumental operating conditions. In the Consolidated mass spectrometer the ion accelerating voltage and the voltage drawing ions from the ion source vary inversely as the mass of the ions, so there will be a larger temperature coefficient for larger values of $m / e$. The small differences in temperature coefficients of sensitivity for $\mathrm{A}^{+}$and $\mathrm{A}^{++}$ and for $\mathrm{A}^{+}$as compared with $\mathrm{N}_{2}^{+}$are accounted for by this mass effect.

The temperature coefficients for molecule ions of most of the hydrocarbons listed in table 3 are much larger than the approximate value of -0.2 percent per degree arising from the changing gas density and collecting efficiency. The experiments suggest that higher temperatures result in an increased probability of excitation to states corresponding to ionization with dissociation rather than to states corresponding to ionization alone. This accounts for the inverse correlation of the temperature coefficients and the relative size of the molecule ion. Furthermore, for stable molecules in which the energy of the excited state corresponding to dissociation is considerably higher than that of the state corresponding to ionization, the temperature coefficient of the molecule ion would be expected to be small, and a large fraction of the total ionization would be contributed by the molecule ion. For benzene and propyne the temperature coefficients are actually slightly less than for monatomic gases (the small difference may not be significant), and the molecule ions are evidently quite stable.

For other molecules, a decrease in the molecule ion peak with increasing temperature must result in an increase in some of the fragment ions, and it is to be expected that the total ionization will have a small negative temperature coefficient comparable to that of a monatomic gas. The data in tables 2 and 3 support this conclusion, for although the spectra are incomplete most of the large peaks are included. Thus, in n-octane if each temperature coefficient of sensitivity is multiplied by the peak height and the sum of these products is divided by the sum of the peaks, the resulting weighted mean temperature coefficient of sensitivity is approximately -0.1 percent per degree. This indicates that the probability of ionization of a molecule is not affected by temperature, but the dissociation of the ions into fragments is temperature sensitive.

The stability of a molecule ion may be measured by the appearance potential of the molecule ion as compared with that of the fragment ions. These potentials are known for a few of the molecules listed in table 3 , and data are given in table 4 . The third column gives the appearance potential of the molecule ion, the fourth column is the lowest appearance potential at which a fragment ion appears. The fifth column is the difference of these two potentials, and it measures the stability of the molecule ion in electron volts. The last column is the temperature coefficient of sensitivity from table 3 . The molecule ion must have an excess of energy equal to or greater than the value in column 5 to dissociate spontaneously and give a fragment ion. The appearance potentials of propyne have not been measured, but the isomer propadiene (allene) has a nearly identical mass spectrum, and it must have nearly the same appearance potential. As appearance potentials are subject to uncertainties of several tenths of a volt, the small differences listed for these compounds are quite uncertain. (The two sets of values for $n$-hexane indicate uncertainties of the same magnitude as the differences.)

TABLE 4. Stability of molecule ions and temperature coefficient of sensitivity

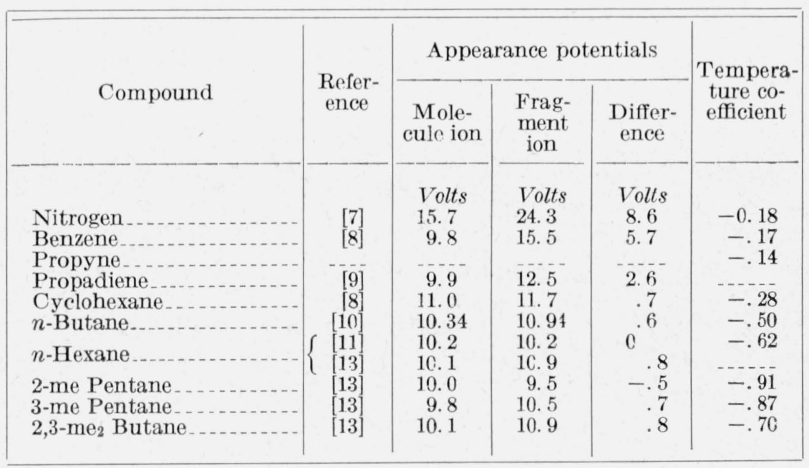

The data indicate that benzene and propyne are quite stable compared to the other hydrocarbons listed, and one would not expect to find appreciable thermal dissociation at $300^{\circ} \mathrm{C}$. This is consistent with the experimental values of the temperature coefficient. Molecule ions of most of the compounds listed in table 3 probably have stabilities of a few tenths of a volt or less. Thus, from the energy 
viewpoint, there is no difficulty in understanding why the heavier hydrocarbons have small molecule ion peaks with a large negative temperature coefficient. It is to be noted, however, that in the mass spectrometer the molecule ions must spontaneously dissociate in less than $10^{-6}$ seconds to be recorded as fragment ions, and the thermal dissociation energy must come from internal vibrational or rotational energy. Ions undergoing any collisions with walls or other molecules are not recorded. It would not be impossible for an ion of negative energy to be observed provided the transition to the dissociated state involved a long half-life compared with $10^{-6}$ seconds. Fox and Langer [12] find that in $n$-butane, fragment ions $\mathrm{C}_{3} \mathrm{H}_{7}{ }^{+}$and $\mathrm{C}_{3} \mathrm{H}_{6}{ }^{+}$appear at the appearance potential of the molecule ion in very low abundance and then increase sharply in abundance at a potential a few tenths of a volt higher. This effect is probably related to the temperature effect discussed in this paper and suggests a serious difficulty in the accurate measurement of appearance potentials.

Berry [4] has pointed out the practical implications of the temperature effect in chemical analysis. Accurate control of the temperature of the ionization chamber is essential for reproducible results, and this is more important for heavy hydrocarbons in the gasoline range than for light gases. In a comparison of results with different instruments, temperature differences can be a more important factor than differences in the other operating conditions. As temperature gradients undoubtedly exist in the ionizachanber, accurate control of the temperature at one point may not be sufficient to insure reproducible spectra. It has been suggested that a better way to maintain reproducibility of spectra is to maintain a constant pattern ratio for the molecule ion of $n$ butane by slight variations in the indicated temperature of the ionization chamber. We have not yet seen experimental evidence, however, to prove that this is better than accurate temperature control at one point.

The authors are grateful to Helen Dean for aiding in the measurement of peak heights in most of the mass spectrograms.

\section{References}

[1] J. A. Hipple, St. Louis meeting of the Am. Inst. Chem. Eng. (Nov. 1944).

[2] R. E. Fox and J. A. Hipple, J. Chem. Phys. 15, 208 (1947).

[3] D. P. Stevenson, J. Chem. Phys. 17, 101 (1949).

[4] C. E. Berry, J. Chem. Phys. 17, 1164 (1949).

[5] V. H. Dibeler and F. Cordero, J. Research NBS 46, (1951) RP2169.

[6] Catalog of mass spectral data, Am. Petroleum Inst. Project 44 .

[7] H. D. Hagstrum and J. T. Tate, Phys. Rev. 59, 354 (1941).

[8] A. Hustrulid, P. Kusch, and J. T. Tate, Phys. Rev. 54, 1037 (1938).

[9] J. Delfosse and W. Bleakney, Phys. Rev. 56, 256 (1939).

[10] D. P. Stevenson and J. A. Hipple, J. Am. Chem. Soc. 64, 1588 (1942).

[11] T. Kambara, J. Phys. Soc. Jap. 1 and 2, 25 and 57 (1946).

[12] R. E. Fox and A. Langer, J. Chem. Phys. 18, 460 (1950).

[13] M. B. Koffel and R. A. Lad, J. Chem. Phys. 16, 420 (1948).

Washington, September 15, 1950. 